\title{
Non-local means based Rician noise filtering for diffusion tensor and kurtosis imaging in human brain and spinal cord
}

Zhongping Zhang ${ }^{1,3 \dagger}$, Dhanashree Vernekar ${ }^{1 \dagger}$, Wenshu Qian ${ }^{1,4}$ and Mina Kim ${ }^{1,2^{*}}$ (1)

\begin{abstract}
Background: To investigate the effect of using a Rician nonlocal means (NLM) filter on quantification of diffusion tensor (DT)- and diffusion kurtosis (DK)-derived metrics in various anatomical regions of the human brain and the spinal cord, when combined with a constrained linear least squares (CLLS) approach.
\end{abstract}

Methods: Prospective brain data from 9 healthy subjects and retrospective spinal cord data from 5 healthy subjects from a 3 T MRI scanner were included in the study. Prior to tensor estimation, registered diffusion weighted images were denoised by an optimized blockwise NLM filter with CLLS. Mean kurtosis (MK), radial kurtosis (RK), axial kurtosis $(A K)$, mean diffusivity (MD), radial diffusivity (RD), axial diffusivity (AD) and fractional anisotropy (FA), were determined in anatomical structures of the brain and the spinal cord. DTI and DKI metrics, signal-to-noise ratio (SNR) and Chisquare values were quantified in distinct anatomical regions for all subjects, with and without Rician denoising.

Results: The averaged SNR significantly increased with Rician denoising by a factor of 2 while the averaged Chisquare values significantly decreased up to $61 \%$ in the brain and up to $43 \%$ in the spinal cord after Rician NLM filtering. In the brain, the mean MK varied from 0.70 (putamen) to 1.27 (internal capsule) while AK and RK varied from 0.58 (corpus callosum) to 0.92 (cingulum) and from 0.70 (putamen) to 1.98 (corpus callosum), respectively. In the spinal cord, FA varied from 0.78 in lateral column to 0.81 in dorsal column while MD varied from $0.91 \times 10^{-3} \mathrm{~mm}^{2} / \mathrm{s}$ (lateral) to $0.93 \times 10^{-3} \mathrm{~mm}^{2} / \mathrm{s}$ (dorsal). RD varied from $0.34 \times 10^{-3} \mathrm{~mm}^{2} / \mathrm{s}$ (dorsal) to $0.38 \times 10^{-3} \mathrm{~mm}^{2} / \mathrm{s}$ (lateral) and AD varied from $1.96 \times 10^{-3} \mathrm{~mm}^{2} / \mathrm{s}$ (lateral) to $2.11 \times 10^{-3} \mathrm{~mm}^{2} / \mathrm{s}$ (dorsal).

Conclusions: Our results show a Rician denoising NLM filter incorporated with CLLS significantly increases SNR and reduces estimation errors of DT- and KT-derived metrics, providing the reliable metrics estimation with adequate SNR levels.

Keywords: Rician noise filter, Diffusion tensor imaging, Diffusion kurtosis imaging, Human brain, Human cervical spinal cord

\section{Background}

Diffusion magnetic resonance imaging (dMRI) is widely

\footnotetext{
*Correspondence: mina.kim@gmail.com

'Zhongping Zhang and Dhanashree Vernekar have contributed equally to this work.

1 Department of Diagnostic Radiology, The University of Hong Kong, Hong Kong SAR, China

Full list of author information is available at the end of the article
}

applied imaging modality allowing to characterize the microstructural properties of brain tissues. Among various kinds of dMRI methods, diffusion tensor imaging (DTI) has been the most commonly used [1-11]. While DTI focuses on the study of white matter (WM) structure providing important information about the tissue anisotropy, diffusion kurtosis imaging (DKI), an extension of DTI provides complementary information especially for

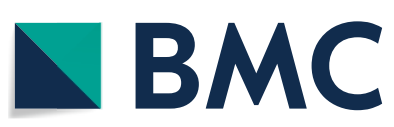

(c) The Author(s) 2021. Open Access This article is licensed under a Creative Commons Attribution 4.0 International License, which permits use, sharing, adaptation, distribution and reproduction in any medium or format, as long as you give appropriate credit to the original author(s) and the source, provide a link to the Creative Commons licence, and indicate if changes were made. The images or other third party material in this article are included in the article's Creative Commons licence, unless indicated otherwise in a credit line to the material. If material is not included in the article's Creative Commons licence and your intended use is not permitted by statutory regulation or exceeds the permitted use, you will need to obtain permission directly from the copyright holder. To view a copy of this licence, visit http://creativecommons.org/licenses/by/4.0/. The Creative Commons Public Domain Dedication waiver (http://creativeco mmons.org/publicdomain/zero/1.0/) applies to the data made available in this article, unless otherwise stated in a credit line to the data. 
analysis of microstructure in gray matter (GM) by probing non-Gaussian diffusion properties [12-16]. In recent years, the interest for DKI has been continuously growing and mean kurtosis (MK), a principal metric of the diffusional non-Gaussianity has demonstrated improved sensitivity in measuring developmental [16-18] and pathological $[15,19]$ changes in neural tissues of both animal and human over conventional DTI. However, one of the challenges in DKI measurement which reduces its practical usage in clinical research is a use of multiple and higher diffusion-weighting (b-values) as compared to a conventional DTI. This results in a low signal-tonoise ratio (SNR) of which the effect plays a large role in yielding the erroneous tensor estimation due to the bias induced by Rician nature of random noise $[20,21]$. The low SNR can also affect the accuracy of diffusion tensor properties such as trace and fractional anisotropy (FA) $[11,22,23]$. Especially, the application of DTI in the spinal cord suffers from the low SNR $[24,25]$ in addition to other challenges such as the small size of the cord, physiological motion, local field inhomogeneity and susceptibility artefacts [26-29].

To date, various denoising methods have been developed to improve the quality of DW images, such as the Gaussian filter [30,31], anisotropic diffusion filter [32-34], linear minimum mean squared error filter [21], random matrix theory [35], multi-shell position-orientation-adaptive smoothing [36] and nonlocal means (NLM) filter [37, 38]. In particular, NLM filter has been suggested to significantly improve MR data quality by reducing Rician noise [20,39-41] and implemented to DTI [42-44] and DKI [45] in the human brain. Moreover, NLM filter has shown to provide efficiency of noise removal while the fine structures and details of images are well preserved [40, 46-51]. Despite considerable research effort in identifying the best denoising algorithm among the existing algorithms for DKI $[45,46,52]$ as well as DTI $[21,37,45,48,53]$, the standard denoising methodology for DKI post-processing part is still to be established, which makes DKI more difficult to use clinically. In order to translate DKI as a clinical tool, it is required to evaluate the direct impact of correcting such bias on regional DKI values associated with SNR in the healthy brain. A few studies investigated inter-subject variability of DKI metrics in the brain of healthy subjects without including noise bias corrections $[54,55]$. To the best of our knowledge, only one study [56] investigated the influence of noise correction by data processing related to inter-subject variability of DKI metrics, however the reported diffusion metrics are limited to mean diffusivity (MD), FA and MK. In DTI of spinal cord, previous studies suggested various correction methods to boost SNR $[25,57-61]$, however the impact of Rician noise filtering in DTI of the human spinal cord has not been reported yet.

In this study, we evaluate the effect of using the Rician NLM filtering in combination with constrained linear least squares (CLLS), one of the least-squares (LS) fitting algorithms commonly used in DTI and DKI studies [13, $62,63]$ of the human brain and spinal cord. In particular, we present here diffusivity and kurtosis measures in different anatomical regions of the healthy human volunteers. Additionally, error measures are quantitatively compared between each DT- and KT-metric with and without implementing a Rician NLM filter (CLLS-R and CLLS, respectively).

\section{Methods}

\section{Subjects}

For brain scans, 9 healthy volunteers ( 5 male, 4 females; mean age $=24 ; \pm$ standard deviation $=2$ years) were recruited through local advertisements. The exclusion criteria for healthy controls were subjects who suffer from any neurological/psychological conditions and any physical disabilities. All participants were studied after signed, informed consent. The study was approved by the local Institutional Review Board. Additionally, the previously obtained spinal cord data including five healthy volunteers ( 1 male, 4 female; mean age $=32 \pm 7$ years) [64] were retrospectively used in the present study for Rician denoising filtering.

\section{Data acquisition}

All MRI scans were performed on a Philips 3 T MRI Achieva scanner (Philips Healthcare, Best, The Netherlands). Brain scans were carried out with a body coil excitation and an 8-channel SENSE head coil for reception. For DKI data, four averaged minimally weighted $\left(b_{0}\right)$ and 32 non-collinear diffusion-encoding directions with two b-values (1000 and $2000 \mathrm{~s} / \mathrm{mm}^{2}$ ) were acquired using single-shot EPI sequence as similar to previous studies [65, 66]. The imaging parameters were: $\mathrm{TR} / \mathrm{TE}=2000 / 69 \mathrm{~ms}$, FOV $=224 \times 224 \mathrm{~mm}^{2}$, acquisition matrix $=88 \times 88$, reconstructed resolution $=2 \times 2 \mathrm{~mm}^{2}$, fold-over direction $=\mathrm{AP}$, EPI factor $=47,44$ axial slices with $3 \mathrm{~mm}$ thickness and no inter-slice gap to cover the whole brain, SENSE factor $=2$, 3/4 partial Fourier encoding, 2 averages and total scan time $=19 \mathrm{~min} .38 \mathrm{~s}$. The number of averages for DWIs was optimized to sustain the reasonable SNR while compromising scan time and six sequential observations of the DWIs with 1 average were obtained in the additional scans for computing the SNR. For anatomical reference, three-dimensional $\mathrm{T}_{1}$-weighted images were also acquired using 3D-MPRAGE sequence with the following parameters: $\mathrm{TR} / \mathrm{TE}=7.0 / 3.2 \mathrm{~ms}$, $\mathrm{TI}=800-818 \quad \mathrm{~ms}, \quad$ FOV $=224 \times 224 \times 167 \mathrm{~mm}^{3}$, 
acquisition matrix $=224 \times 224$, reconstruction resolution $=1 \times 1 \mathrm{~mm}^{2}$, 167 sagittal slices with $1 \mathrm{~mm}$ thickness and no inter-slice gap to cover the whole brain, 1 average and total scan time $=10 \mathrm{~min} .41 \mathrm{~s}$. Acquisition parameters for spinal cord DTI data can be found from the previous study [64] which included four averaged minimally weighted $\left(\mathrm{b}_{0}\right)$ and 15 diffusion-weighted volumes $\left(\mathrm{b}\right.$-value $=500 \mathrm{~s} / \mathrm{mm}^{2}$ ) using single-shot echo-planar-imaging (EPI) sequence. Two imaging stacks in axial plane covered upper ( $\mathrm{C} 1$ to $\mathrm{C} 3$ ) and lower ( $\mathrm{C} 4$ to $\mathrm{C} 6$ ) portions of the cervical spinal cord (slice thickness $=2.5$ and $5 \mathrm{~mm}$, respectively).

\section{Data postprocessing}

Data were analysed using a custom-written program in Matlab (Mathworks, Natick, MA) and the DKE software (https://www.nitrc.org/projects/dke/) [52]. Detailed data postprocessing procedures for DKI are illustrated in Fig. 1. All DWIs were co-registered to the initial $b_{0}$ image using a 6-degree-of-freedom, rigid body transformation procedure supplied in automated image registration (AIR) [67] in order to reduce the impact of motion artefact and eddy current distortion. Each gradient direction was revised with respect to transformation matrix after registration. Prior to tensor estimation, registered DWIs were denoised by an optimized blockwise NLM filter consisting of a blockwise implementation with automatic tuning of the smoothing parameter and block selection as described elsewhere [39, 40]. The numerical value for SNR in regions of interest (ROIs) for baseline image $(b=0)$ was computed by the signal statistics in a difference image as described in Farrell et al. [68]. DT and KT were estimated using CLLS algorithm suggested by Tabesh et al. [52] based on the definition of all DT- and KT-metrics [10, 14, 52].

\section{ROI-based measurements}

For brain images, 6 ROIs including putamen (PUT; averaged area size $=985 \mathrm{~mm}^{2}$ ), globus pallidus (GP; averaged area size $\left.=525 \mathrm{~mm}^{2}\right)$, corpus callosum $(C C$; averaged area size $=2497 \mathrm{~mm}^{2}$ ), internal capsule (IC; averaged area size $=1643 \mathrm{~mm}^{2}$ ), external capsule (EC; averaged area size $\left.=1241 \mathrm{~mm}^{2}\right)$ and cingulum $(\mathrm{Cg}$; averaged area size $=678 \mathrm{~mm}^{2}$ ) were manually drawn in a FA map and a color-coded map using ImageJ (National Institutes of Health, Bethesda, MD) by a trained rater (Z.Z.) referencing to the standard human brain atlas (Fig. 2) [69]. All 6 ROIs were then transferred to the rest of the DT- and KT-derived maps in order to compute the average and error measures within the ROIs for each subject. Chisquare value $\left(\chi^{2}\right)$ was calculated by computing the difference between the observed and estimated signals to estimate the goodness of fitting. Later, another trained rater (W.Q.) independently placed ROIs on all datasets to ensure the indices were reliable across raters.

For spinal cord images, 6 slices matching $\mathrm{C} 1$ to $\mathrm{C} 6 \mathrm{lev}$ els of the spinal cord in a FA map per each subject, were selected around mid-level of two intervertebral discs to minimize susceptibility artefact typically caused near the disc-tissue boundaries. In each chosen slice, 3 ROIs were manually placed in the lateral left and right, and dorsal columns (Fig. 4a), a couple of pixels away from

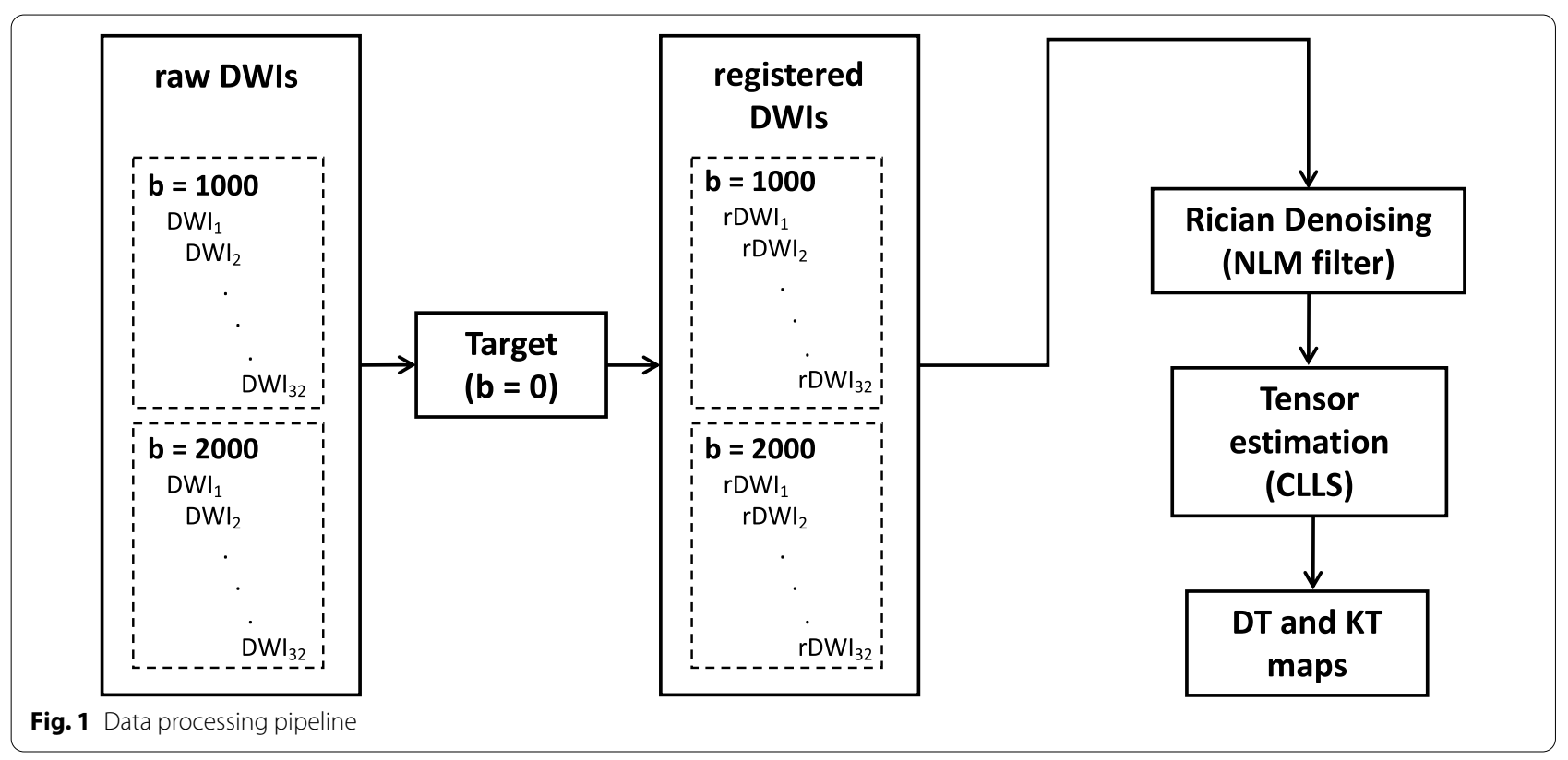



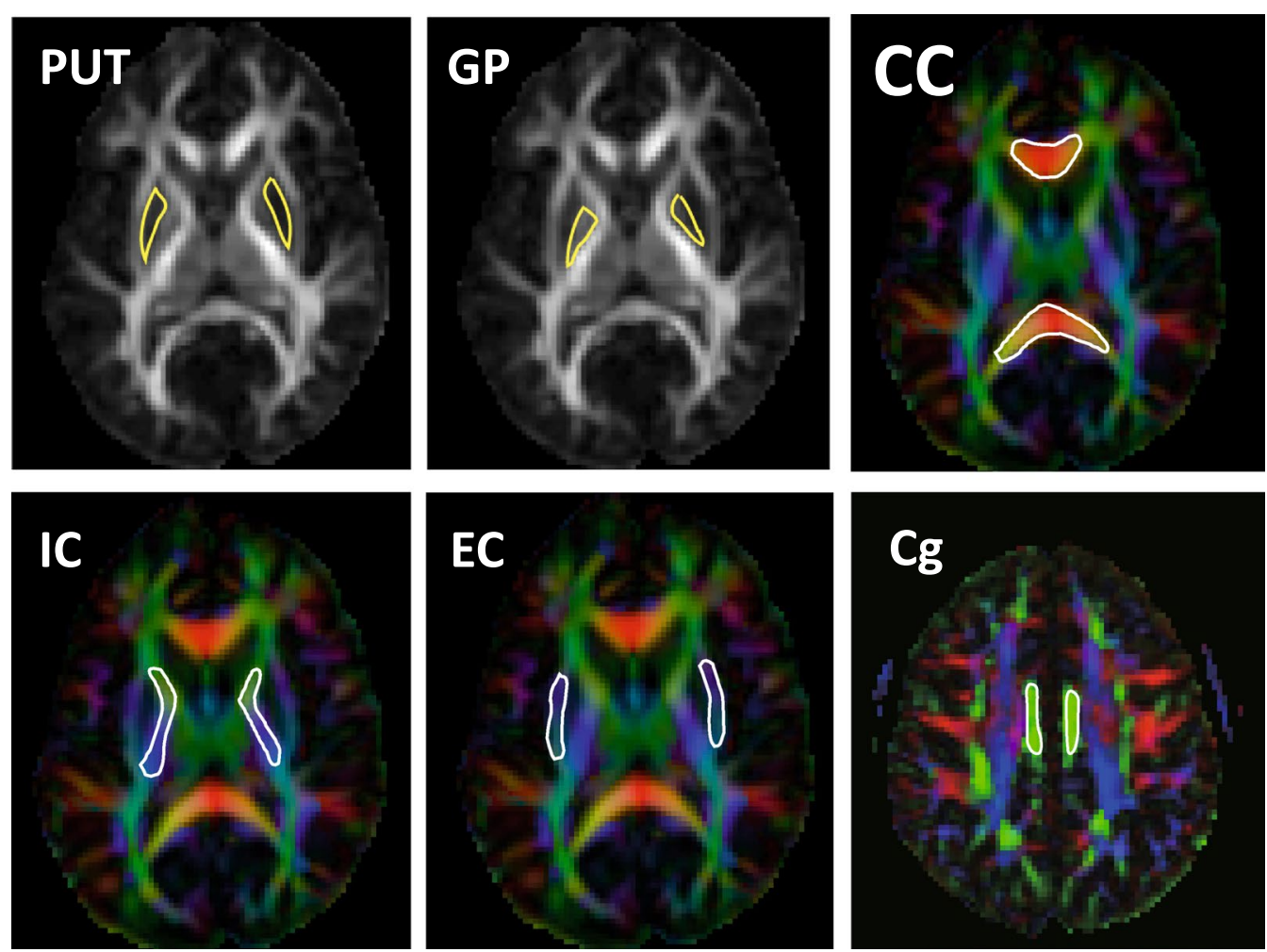

Fig. 2 Location of ROIs on the FA and color-coded maps of the brain. Abbreviations PUT, putamen; GP, globus pallidus; CC, corpus callosum; IC, internal capsule; EC, external capsule; Cg, cingulum

the boundaries of GM/WM and WM/cerebrospinal fluid (CSF) to avoid partial volume effect using Image J (National Institutes of Health, Bethesda, MD, USA). The area size of $1.563 \mathrm{~mm}^{2}$ for each ROI was consistent across all subjects. These ROIs were then transferred to the rest of the DTI-derived maps including mean FA, MD, radial diffusivity (RD) and axial diffusivity (AD), in order to compute the average of DTI-indices within the ROIs for each subject. $X^{2}$ was calculated in a voxel-by-voxel basis to evaluate the goodness of denoising performance. SNR and DTI-derived metrics were recalculated without the prior-denoising process and using the same ROIs that were previously defined for comparison.

\section{Statistical analysis}

To evaluate the inter-rater reliability, the intraclass correlation coefficient (ICC) was computed to assess reliability of mean values measured by two raters for each ROI as described by Shrout and Fleiss [70]. Averaged SNR values of $b_{0}$ images and ROI-based diffusion and kurtosis metrics were compared between CLLS and CLLS-R by paired Mann-Whitney $U$ test. All statistical analyses were performed using commercially available software (SPSS, Chicago, IL, USA). P-values of 0.05 were considered to be significant.

\section{Results}

In a single $\mathrm{b}_{0}$ image of the brain, SNR ranged from 10.85 (GP) to 20.50 (EC) with CLLS (without Rician NLM filtering) and from 25.25 (CC) to 74.38 (EC) with CLLS-R (with Rician NLM filtering) (Table 1). SNR significantly increased in all 6 ROIs using CLLS-R $(244 \%, 177 \%, 62 \%$, $162 \%, 263 \%$ and $125 \%$ for PUT, GP, CC, IC, EC and Cg,

Table 1 Comparison of averaged SNR \pm standard deviation of $b_{0}$ images in the brain of healthy volunteers $(n=9)$ measured using CLLS and CLLS-R. Paired MannWhitney $U$ test was used in the comparison between CLLS and CLLS-R

\begin{tabular}{llll}
\hline & CLLS & CLLS-R & $\boldsymbol{p}$ \\
\hline PUT & $17.65 \pm 0.73$ & $60.75 \pm 6.27$ & $<0.05$ \\
GP & $10.85 \pm 0.62$ & $30.02 \pm 4.81$ & $<0.05$ \\
CC & $15.61 \pm 1.19$ & $25.25 \pm 2.54$ & $<0.05$ \\
IC & $15.78 \pm 0.28$ & $41.30 \pm 3.88$ & $<0.05$ \\
EC & $20.50 \pm 1.14$ & $74.38 \pm 4.39$ & $<0.05$ \\
Cg & $19.56 \pm 2.23$ & $44.04 \pm 4.42$ & $<0.05$ \\
\hline
\end{tabular}

SNR, signal-to-noise ratio; PUT, putamen; GP, globus pallidus; CC, corpus callosum; IC, internal capsule; EC, external capsule; $\mathrm{Cg}$, cingulum 
respectively with $p<0.05$ for all ROIs) as compared to CLLS. In upper spinal cord levels C1-C3 (2.5 mm slice thickness), SNR ranged from 9.77 (Lateral) to 10.66 (Dorsal) with CLLS and from 16.32 (Lateral) to 21.97 (Dorsal) with CLLS-R (Table 2). In lower spinal cord levels C4-C6 (5 mm slice thickness), SNR ranged from 23.02 (Dorsal) to 23.73 (Lateral) with CLLS and from 45.21 (Dorsal) to 49.23 (Lateral) with CLLS-R (Table 2). In C1-C3, mean SNR significantly increased by $67.09 \%$ and $106.11 \%$ for lateral and dorsal respectively $(p<0.001$ for both columns). Similarly, significant increase of mean SNR by $107.50 \%$ and $96.38 \%$ was observed for lateral and dorsal respectively in C4-C6 ( $p<0.001$ for both columns).

The $X^{2}$ maps in the brain of a representative subject (25, male) (Fig. 3a) and group differences (Fig. 3b) demonstrate that $\chi^{2}$ values estimated by CLLS-R are decreased in deep GM, WM and overall cortical GM as compared to those by CLLS. Group comparison averaged across 9 subjects at each ROI shows that mean $\chi^{2}$ values significantly decreased in all 6 regions of brain using CLLS-R as compared to CLLS (32-61\% with $p<0.05)$. Similarly, the averaged $\chi^{2}$ values of the spinal cord over 5 healthy subjects significantly decreased by maximum $43 \%$ with Rician denoising as compared those without Rician denoising in all 3 columns throughout $\mathrm{C} 1-\mathrm{C} 6(p<0.001)$ (Fig. 4B). In the representative DT- and KT-derived brain maps, KT-derived metrics (MK, axial kurtosis (AK) and radial kurtosis (RK)) show visual difference between CLLS and CLLS-R (Fig. 5). Kurtosis maps (AK and RK) using CLLS-R illustrate significant decrease in a number of erroneous pixels as compared to those using CLLS while moderate improvement is noticeable in MK maps (Fig. 5).

All the KT-derived metrics (MK, RK, and AK), together with the DT-derived metrics (FA, MD, AD, and RD) by CLLS-R averaged across all subjects at each ROI are listed in Table 3. In the brain, MK varied from 0.70 to 1.27 with the lowest MK value obtained in PUT and highest in IC while RK varied from 0.70 (PUT) to 1.98 (CC). AK varied from 0.58 in the CC to 0.92 in $\mathrm{Cg}$. FA varied from 0.12 to 0.65 , with the lowest FA value obtained in the PUT and highest in CC while MD varied from $0.75 \times 10^{-3} \mathrm{~mm}^{2} / \mathrm{s}$ (PUT) to

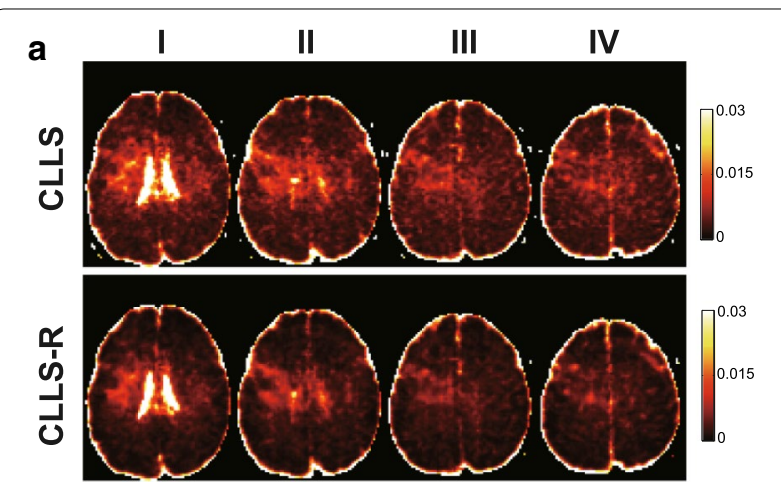

b

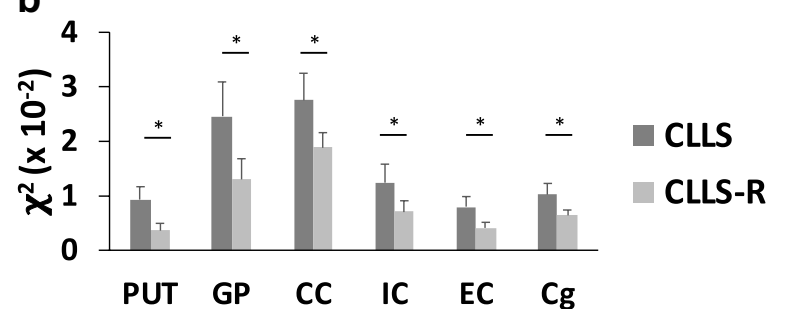

Fig. 3 a Representative 4 neighbouring axial slices of $x^{2}$ maps in a healthy subject ( 25 years old, male) using CLLS and CLLS-R. b Group differences of the $x^{2}$ measurement at each brain region between before- (CLLS) and after-Rician denoising (CLLS-R). Significant differences between groups are indicated by ${ }^{*} p<0.05$. Abbreviations PUT, putamen; GP, globus pallidus; CC, corpus callosum; IC, internal capsule; EC, external capsule; Cg, cingulum

$1.04 \times 10^{-3} \mathrm{~mm}^{2} / \mathrm{s}(\mathrm{CC}) . \mathrm{RD}$ varied from $0.50 \times 10^{-3}$ $\mathrm{mm}^{2} / \mathrm{s}$ in IC to $0.71 \times 10^{-3} \mathrm{~mm}^{2} / \mathrm{s}$ in PUT and AD varied from $0.85 \times 10^{-3} \mathrm{~mm}^{2} / \mathrm{s}$ in PUT to $1.95 \times 10^{-3}$ $\mathrm{mm}^{2} / \mathrm{s}$ in CC. ICC of DT- and KT-indices measured in various ROIs by two raters ranged from 0.744 to 0.999 (Table 4). In the spinal cord, FA varied from 0.78 in lateral column to 0.81 in dorsal column while MD varied from $0.91 \times 10^{-3} \mathrm{~mm}^{2} / \mathrm{s}$ (lateral) to $0.93 \times 10^{-3}$ $\mathrm{mm}^{2} / \mathrm{s}$ (dorsal). RD varied from $0.34 \times 10^{-3} \mathrm{~mm}^{2} / \mathrm{s}$ (dorsal) to $0.38 \times 10^{-3} \mathrm{~mm}^{2} / \mathrm{s}$ (lateral) and $\mathrm{AD}$ varied from $1.96 \times 10^{-3} \mathrm{~mm}^{2} / \mathrm{s}$ (lateral) to $2.11 \times 10^{-3}$ $\mathrm{mm}^{2} / \mathrm{s}$ (dorsal). DT- and KT-derived metrics estimated using CLLS-R were significantly different from those by CLLS in most of examined anatomical regions

Table 2 Mean \pm standard deviation of averaged SNR of $b_{0}$ images in 5 healthy volunteers in lateral \& dorsal columns before and after denoising

\begin{tabular}{|c|c|c|c|c|c|c|}
\hline & \multicolumn{3}{|l|}{ Up (C1-C3) } & \multicolumn{3}{|l|}{ Low (C4-C6) } \\
\hline & CLLS & CLLS-R & $p$ & CLLS & CLLS-R & $p$ \\
\hline Lateral & $9.77 \pm 0.47$ & $16.32 \pm 3.72$ & $<0.001$ & $23.73 \pm 1.24$ & $49.23 \pm 5.33$ & $<0.001$ \\
\hline Dorsal & $10.66 \pm 0.71$ & $21.97 \pm 3.77$ & $<0.001$ & $23.02 \pm 0.56$ & $45.21 \pm 7.03$ & $<0.001$ \\
\hline
\end{tabular}

The right and left lateral columns have been averaged together for this analysis. Paired Mann-Whitney U test was used in the comparison between CLLS and CLLS-R 


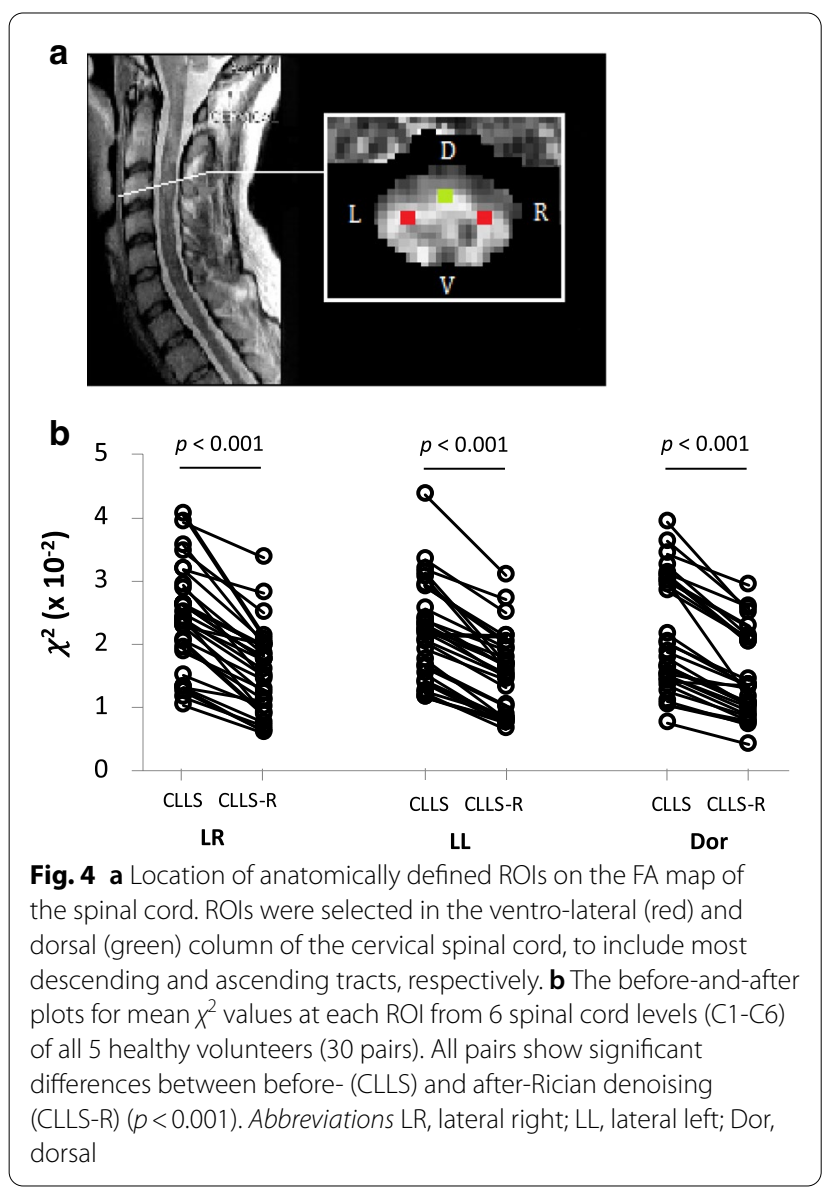

(Additional File 1: Supporting Information Table S1). The mean FA values using CLLS-R were significantly decreased in all 6 regions $(3-11 \%$ with $p<0.05)$ compared to CLLS. Similar results were found for AD with exception of the PUT, CC and Cg. Meanwhile MD, RD, RK and MK values were significantly increased with exception of the PUT, IC and EC for MD and PUT for RD, CC and Cg for RK and PUT for MK. Additionally, the ROI-based mean $\chi^{2}$ values showed that CLLS-R significantly reduced $\chi^{2}$ values over all ROIs compared to CLLS (32-61\% with $p<0.05)$.

\section{DISCUSSION}

Despite great deal of effort in comparing various denoising algorithms for DKI in previous studies [45, 46, 52] and inter-subject variability of DKI metrics in brain of healthy subjects $[54,55]$, the direct impact of Rician noise correction on DKI data has not been sufficiently studied, especially related to improvement on SNR and error measures in various anatomical regions. In this study, we evaluate the influence of the Rician NLM filter in combination with CLLS algorithm on the brain DKI and the spinal cord DTI data. Rician NLM filter in combination with CLLS algorithm has the potential to be useful in clinical research because it is easy and efficient to implement. To the best of our knowledge, this is the first study to assess the influence of combining the NLM filter with CLLS in DTI of the spinal cord and DKI in the brain and to report ROI-based changes in SNR and the associated error estimates. To compare diffusional measures using different processing methods (CLLS and CLLS-R), we obtained whole brain DKI data from 9 healthy volunteers and compared mean DT- and KT-derived indices using CLLS and CLLS-R. Rician NLM filter was also applied to the previously acquired spinal cord data retrospectively [64].

\section{Measurement of SNR}

SNR assessment is important for reliable quantification of diffusional metrics [71]. When SNR is low, Rician noise does not only cause random fluctuations but also a signal dependent bias to the data, which may lead to difficulty in postprocessing such as tensor calculation. However, SNR levels are not routinely reported although previous reports suggest that low SNR causes a bias in FA which may vary with numerous other technical factors such as the region of brain being studied, field strength, hardware and software $[68,72,73]$. As a first step to evaluate the influence of Rician denoising, we have measured SNR in various anatomical areas of the brain and the spinal cord with and without Rician denoising. Our results show that Rician NLM filtering yields the significant increase of SNR in the brain DKI and the spinal cord DTI data (Table 1 and 2). The ROI-based SNR values with noise correction in our study are consistent with the previous study by Seo et al. [74] which has reported SNR thresholds 20 in the CC and 70 in the PUT for biasfree estimation of tensor metrics. It should be noted that those SNR values from the literatures were obtained from different acquisition protocols. Thus, when comparing those values with literature values, care should be taken to ensure the similarity of protocol chosen for comparison. For instance, the eddy current and off-resonance effects in a DWI sequence may substantially vary with b-value and diffusion gradient direction. Additionally, the spatial noise distribution can be varied by coil geometry, phase-encoding direction and acceleration factor of parallel imaging $[38,75,76]$ which also need to be taken into account for. In our study, it is also observed that the degree of SNR improvement relates to the underlying structures. For instance, the CC has the smallest SNR increase among other regions, which might be attributed to the previous findings that the noise within accelerated images is nonhomogeneous with higher signal peripherally and noise centrally when parallel imaging is used [77-79]. As the levels and spatial distributions of noise 


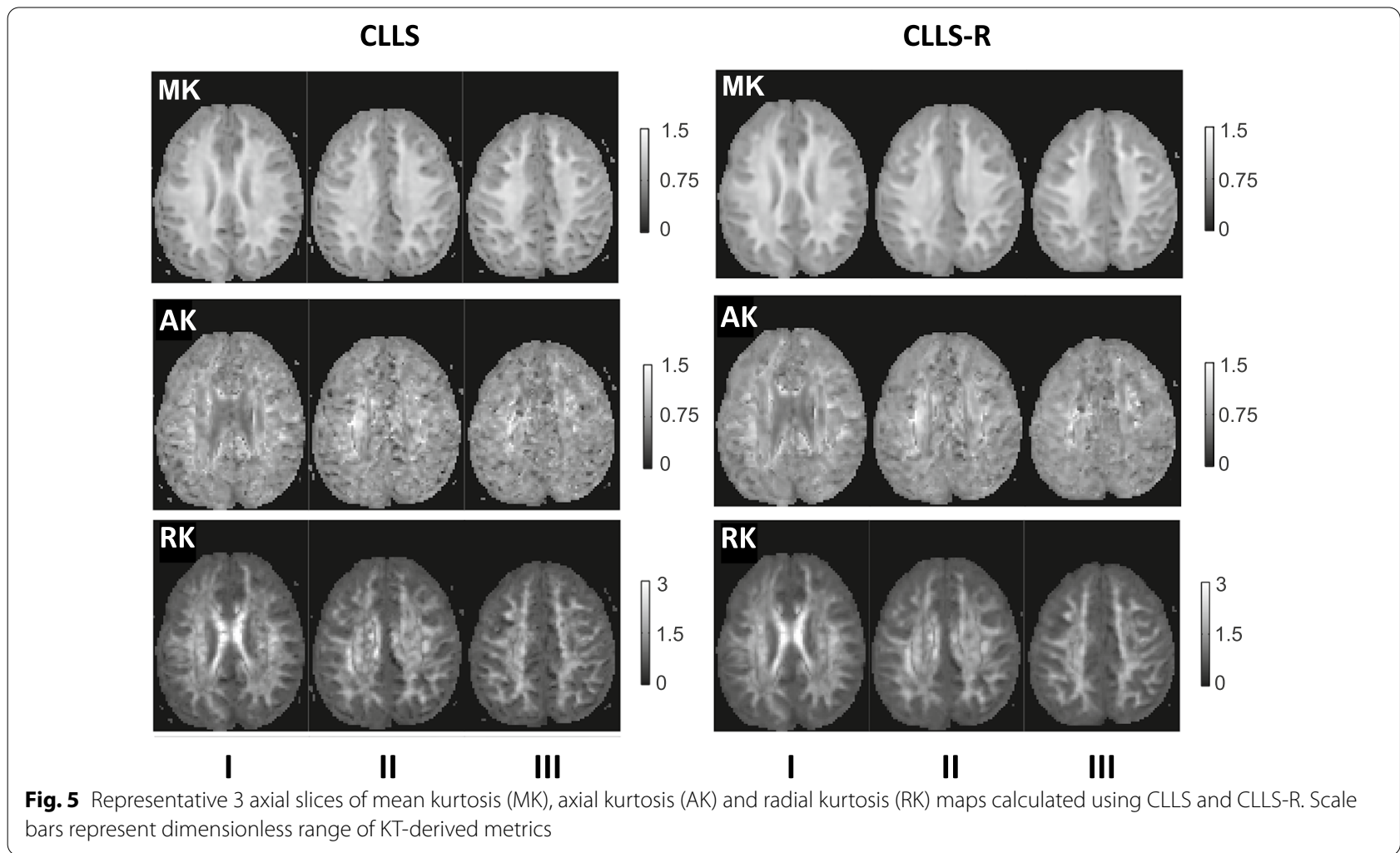

Table 3 ROI-based mean \pm standard deviation of DT- and KT-indices averaged across all subjects at each ROI in the brain and the spinal cord using CLLS-R

\begin{tabular}{|c|c|c|c|c|c|c|c|}
\hline & MK & RK & AK & $\mathrm{MD}\left(10^{-3} \mathrm{~mm}^{2} / \mathrm{s}\right)$ & FA & $\mathrm{RD}\left(10^{-3} \mathrm{~mm}^{2} / \mathrm{s}\right)$ & $\mathrm{AD}\left(10^{-3} \mathrm{~mm}^{2} / \mathrm{s}\right)$ \\
\hline \multicolumn{8}{|c|}{ Brain } \\
\hline PUT & $0.70 \pm 0.04$ & $0.70 \pm 0.04$ & $0.72 \pm 0.04$ & $0.75 \pm 0.03$ & $0.12 \pm 0.02$ & $0.71 \pm 0.02$ & $0.85 \pm 0.04$ \\
\hline GP & $1.03 \pm 0.06$ & $1.13 \pm 0.09$ & $0.89 \pm 0.08$ & $0.78 \pm 0.04$ & $0.26 \pm 0.02$ & $0.68 \pm 0.03$ & $0.99 \pm 0.06$ \\
\hline CC & $1.09 \pm 0.04$ & $1.98 \pm 0.10$ & $0.58 \pm 0.02$ & $1.04 \pm 0.05$ & $0.65 \pm 0.02$ & $0.58 \pm 0.05$ & $1.95 \pm 0.07$ \\
\hline $\mathrm{IC}$ & $1.27 \pm 0.01$ & $1.94 \pm 0.05$ & $0.71 \pm 0.03$ & $0.82 \pm 0.02$ & $0.59 \pm 0.02$ & $0.50 \pm 0.02$ & $1.44 \pm 0.05$ \\
\hline EC & $0.88 \pm 0.04$ & $1.14 \pm 0.06$ & $0.72 \pm 0.03$ & $0.82 \pm 0.02$ & $0.32 \pm 0.03$ & $0.68 \pm 0.02$ & $1.11 \pm 0.05$ \\
\hline $\mathrm{Cg}$ & $0.99 \pm 0.03$ & $1.51 \pm 0.16$ & $0.92 \pm 0.02$ & $0.86 \pm 0.03$ & $0.42 \pm 0.04$ & $0.65 \pm 0.04$ & $1.29 \pm 0.07$ \\
\hline \multicolumn{8}{|c|}{ Spinal Cord } \\
\hline LR & - & - & - & $0.91 \pm 0.05$ & $0.78 \pm 0.09$ & $0.38 \pm 0.06$ & $1.96 \pm 0.05$ \\
\hline$L L$ & - & - & - & $0.93 \pm 0.07$ & $0.79 \pm 0.08$ & $0.38 \pm 0.05$ & $2.01 \pm 0.09$ \\
\hline Dor & - & - & - & $0.93 \pm 0.04$ & $0.81 \pm 0.07$ & $0.34 \pm 0.03$ & $2.11 \pm 0.08$ \\
\hline
\end{tabular}

PUT, putamen; GP, globus pallidus; CC, corpus callosum; IC, internal capsule; EC, external capsule; Cg, cingulum; LR, lateral right; LL, lateral left; Dor, dorsal

are not an equal across the DW image, it is expected that various anatomical areas with different SNR requirements have diverse range of SNR increase rate.

While denoising DTI with low SNR addressing the strong influence of Rician bias in the brain has been well presented by different studies [48, 80, 81], Rician NLM denoising has not been established in the spinal cord DTI where most of previous studies have focused on data acquisition or motion correction methods to improve SNR $[25,27,58,82]$. The mean SNR values without Rician denoising in C1-C3 in our study are within a range of values from a multi-centre study reported by Samson et al. (6.74 - 10.9) [27]. However, our mean SNR values significantly increased in both lateral and dorsal areas of the spinal cord levels C1-C6 after a Rician denoising (Table 2). Our results indicate 
Table 4 Intraclass correlation coefficient of DT- and KT-indices measured in various ROIs by two raters ${ }^{\mathrm{a}}$

\begin{tabular}{llllllll}
\hline & MK & RK & AK & MD & FA & RD & AD \\
\hline PUT & 0.911 & 0.749 & 0.959 & 0.971 & 0.856 & 0.985 & 0.947 \\
GP & 0.893 & 0.914 & 0.893 & 0.956 & 0.768 & 0.930 & 0.905 \\
GM $^{b}$ & 0.992 & 0.991 & 0.968 & 0.967 & 0.988 & 0.963 \\
CC & 0.949 & 0.974 & 0.962 & 0.896 & 0.906 & 0.906 & 0.980 \\
IC & 0.857 & 0.910 & 0.792 & 0.974 & 0.744 & 0.892 \\
EC & 0.910 & 0.823 & 0.968 & 0.987 & 0.926 & 0.911 \\
Cg & 0.938 & 0.971 & 0.834 & 0.995 & 0.982 & 0.992 & 0.884 \\
WMc & 0.997 & 0.995 & 0.991 & 0.992 & 0.996 & 0.985 \\
All ROls & 0.996 & 0.997 & 0.988 & 0.994 & 0.998 & 0.990 \\
\hline
\end{tabular}

PUT, putamen; GP, globus pallidus; CC, corpus callosum; IC, internal capsule; EC, external capsule; Cg, cingulum; GM, gray matter; WM, white matter

a The $p$ value is $<0.001$ for all metrics in all ROls

b GM includes PUT and GP

c WM includes CC, IC, EC and Cg

that a simple noise correction method in the spinal cord such as Rician denoising being used in our study increases SNR (Table 2) and thereby can reduce error estimates (Fig. 4b). This is not surprising given the improved quantification of dMRI in the brain, however worth reporting for the growing need of the spinal cord DTI in the clinical research despite more intrinsic challenges in the spinal cord as compared to the brain.

\section{Estimation errors}

Significantly reduced number of erroneous pixels (black holes) was observed in AK, RK and MK using CLLS-R as compared to CLLS in the brain (Fig. 5). In particular, clear difference is observed in cortical GM, where the voxel values with the erroneously estimated tensor are lower using CLLS as compared to CLLS-R (Fig. 3a). Note that voxels with extreme (negative or zero tensor) values were excluded from the computation. Even after all of extremely erroneous voxels were removed, the ROI-based mean $\chi^{2}$ values significantly decreased over all ROIs when estimated using CLLS-R compared to CLLS in all examined anatomical regions (Fig. 3b), suggesting the impact of Rician denoising step towards KT estimation in the brain. Additionally, the mean $X^{2}$ values in the spinal cord show that Rician NLM filtering significantly reduced error estimates in both lateral and dorsal columns (Fig. 4b). Reduced $\chi^{2}$ values both in the brain and the spinal cord, imply that accuracy of tensor estimation is significantly improved with Rician NLM filtering (Figs. 3b and $4 \mathrm{~b}$ ).

\section{Regional values of DT- and KT-derived metrics}

Currently, a few reports in the literature are available for comparison with our results [54-56, 83, 84]. Table 5 provides an overview of those works in the literature that include values of parameter estimates. While CLLS estimation led to overestimated DT-derived metrics, especially in FA (Additional File 1: Supporting Information Table S1), which is a common problem in human DTI studies $[68,85,90]$, the DT- and KT-derived metrics using CLLS-R are consistent with some of the previously reported values (Table 3). Nevertheless, it is observed that the discrepancy in diffusional metrics values exists amongst various studies in the literature. There are a few factors that may explain discrepancy between studies, from data acquisition to postprocessing perspective. In DTI, it has been well known that low SNR causes a bias in DT-derived metrics, leading to overestimation of FA [68, 72, 73]. When SNR is low, Rician noise does not only cause random fluctuations but also a signal dependent bias to the data, which may lead to difficulty in postprocessing such as tensor calculation. Therefore bias-free measurements require adequate SNR [85] and DTI studies often report SNR values to assure the reliability of the estimated metrics. However, SNR levels are not routinely reported in DKI, resulting in the challenges of comparing results between studies. Therefore it is desirable to ensure the DT- and KT-derived indices are estimated with adequate SNR levels before the comparison between studies. Our results show that the mean MK varied from 0.70 (PUT) to 1.27 (IC) while AK and RK varied from $0.58(\mathrm{CC})$ to $0.92(\mathrm{Cg})$ and from 0.70 (PUT) to $1.98(\mathrm{CC})$, respectively, with a range of SNR levels (from 25.25 in CC to 60.75 in PUT) in various anatomical structures of the brain. Additionally, inter-subject differences substantially 
Table 5 Regional values in the healthy brain from the literature

\begin{tabular}{|c|c|c|c|c|c|c|c|c|}
\hline Region & References & $\begin{array}{l}\text { Number of directions (DIR), } \\
\text { b-values }\left(\mathrm{ms} / \mu \mathrm{m}^{2}\right)\end{array}$ & Voxel size $\left(\mathrm{mm}^{3}\right)$ & MK & RK & $\mathrm{MD}\left(\mathrm{mm}^{2} / \mathrm{s}\right)$ & FA & $\mathrm{RD}\left(\mathrm{mm}^{2} / \mathrm{s}\right)$ \\
\hline PUT & {$[54]$} & $\begin{array}{l}15 \text { DIR, } 5 \text { b-values }(0,500,1000 \\
2500,2750)\end{array}$ & $2 \times 2 \times 2$ & $0.67 \pm 0.08$ & $0.61 \pm 0.08$ & $0.79 \pm 0.03$ & $0.15 \pm 0.02$ & $0.73 \pm 0.03$ \\
\hline PUT & {$[55]$} & 50 DIR, 3 b-values $(0,1000,2000)$ & $1.9 \times 1.9 \times 5$ & $0.77 \pm 0.01$ & $0.85 \pm 0.01$ & $1.72 \pm 0.01$ & $0.16 \pm 0.01$ & $1.57 \pm 0.03$ \\
\hline GP & [54] & $\begin{array}{l}15 \text { DIR, } 5 \text { b-values }(0,500,1000 \text {, } \\
2500,2750)\end{array}$ & $2 \times 2 \times 2$ & $1.06 \pm 0.08$ & $1.05 \pm 0.10$ & $0.86 \pm 0.08$ & $0.27 \pm 0.04$ & $0.74 \pm 0.06$ \\
\hline $\begin{array}{l}\text { GP (Left) } \\
\text { GP (Right) }\end{array}$ & {$[56]$} & 60 DIR, 3 b-values $(0,1000,2800)$ & $2.2 \times 2.2 \times 2.2$ & $\begin{array}{l}1.76 \pm 0.15 \\
1.85 \pm 0.17\end{array}$ & - & - & - & - \\
\hline CC (genu) & [54] & $\begin{array}{l}15 \text { DIR, } 5 \text { b-values }(0,500,1000 \\
2500,2750)\end{array}$ & $2 \times 2 \times 2$ & $1.06 \pm 0.11$ & $2.07 \pm 0.45$ & $0.93 \pm 0.06$ & $0.80 \pm 0.04$ & $0.36 \pm 0.07$ \\
\hline CC (genu) & {$[55]$} & 50 DIR, 3 b-values $(0,1000,2000)$ & $1.9 \times 1.9 \times 5$ & $0.90 \pm 0.05$ & $0.90 \pm 0.07$ & $1.82 \pm 0.08$ & $0.70 \pm 0.05$ & $1.04 \pm 0.07$ \\
\hline CC (genu) & [83] & 64 DIR, 3 b-values $(0,1000,2000)$ & $2.5 \times 2.5 \times 2.5$ & $0.94 \pm 0.07$ & - & $1.38 \pm 0.12$ & $0.44 \pm 0.04$ & - \\
\hline CC (splenium) & [54] & $\begin{array}{l}15 \text { DIR, } 5 \text { b-values }(0,500,1000 \\
2500,2750)\end{array}$ & $2 \times 2 \times 2$ & $1.32 \pm 0.09$ & $2.72 \pm 0.41$ & $0.89 \pm 0.09$ & $0.83 \pm 0.03$ & $0.31 \pm 0.07$ \\
\hline CC (splenium) & {$[55]$} & 50 DIR, 3 b-values $(0,1000,2000)$ & $1.9 \times 1.9 \times 5$ & $1.07 \pm 0.08$ & $1.05 \pm 0.07$ & $1.70 \pm 0.06$ & $0.76 \pm 0.04$ & $0.87 \pm 0.03$ \\
\hline CC (splenium) & {$[83]$} & 64 DIR, 3 b-values $(0,1000,2000)$ & $2.5 \times 2.5 \times 2.5$ & $1.14 \pm 0.09$ & - & $1.17 \pm 0.10$ & $0.54 \pm 0.05$ & - \\
\hline IC & {$[84]$} & $\begin{array}{l}15 \text { DIR, } 6 \text { b-values }(0,500,1000 \\
1500,2000,2500)\end{array}$ & $2 \times 2 \times 2$ & $1.05 \pm 0.08$ & $0.84 \pm 0.03$ & - & - & - \\
\hline $\begin{array}{l}\text { IC (Left) } \\
\text { IC (Right) }\end{array}$ & {$[56]$} & 60 DIR, 3 b-values $(0,1000,2800)$ & $2.2 \times 2.2 \times 2.2$ & $\begin{array}{l}1.45 \pm 0.06 \\
1.49 \pm 0.07\end{array}$ & - & - & - & - \\
\hline ALIC & {$[54]$} & $\begin{array}{l}15 \text { DIR, } 5 \text { b-values }(0,500,1000 \\
2500,2750)\end{array}$ & $2 \times 2 \times 2$ & $1.04 \pm 0.10$ & $1.60 \pm 0.28$ & $0.87 \pm 0.05$ & $0.60 \pm 0.04$ & $0.53 \pm 0.05$ \\
\hline PLIC & [54] & $\begin{array}{l}15 \text { DIR, } 5 \text { b-values }(0,500,1000 \\
2500,2750)\end{array}$ & $2 \times 2 \times 2$ & $1.23 \pm 0.09$ & $2.04 \pm 0.23$ & $0.89 \pm 0.09$ & $0.71 \pm 0.04$ & $0.45 \pm 0.07$ \\
\hline EC & [54] & $\begin{array}{l}15 \text { DIR, } 5 \text { b-values }(0,500,1000 \\
2500,2750)\end{array}$ & $2 \times 2 \times 2$ & $0.81 \pm 0.05$ & $1.02 \pm 0.09$ & $0.90 \pm 0.05$ & $0.41 \pm 0.03$ & $0.70 \pm 0.04$ \\
\hline EC & {$[55]$} & 50 DIR, 3 b-values $(0,1000,2000)$ & $1.9 \times 1.9 \times 5$ & $0.85 \pm 0.01$ & $0.93 \pm 0.05$ & $1.73 \pm 0.19$ & $0.38 \pm 0.03$ & $1.28 \pm 0.03$ \\
\hline $\mathrm{Cg}$ & [54] & $\begin{array}{l}15 \text { DIR, } 5 \text { b-values }(0,500,1000 \\
2500,2750)\end{array}$ & $2 \times 2 \times 2$ & $1.07 \pm 0.07$ & $1.85 \pm 0.26$ & $0.86 \pm 0.07$ & $0.66 \pm 0.06$ & $0.48 \pm 0.08$ \\
\hline $\mathrm{Cg}$ & {$[55]$} & 50 DIR, 3 b-values $(0,1000,2000)$ & $1.9 \times 1.9 \times 5$ & $0.94 \pm 0.03$ & $0.96 \pm 0.07$ & $1.64 \pm 0.05$ & $0.55 \pm 0.05$ & $1.08 \pm 0.05$ \\
\hline
\end{tabular}

PUT, putamen; GP, globus pallidus; CC, corpus callosum; IC, internal capsule; ALIC, anterior limb of IC; PLIC, posterior limb of IC; EC, external capsule; Cg, cingulum

contribute the within-group variability [86], and may partially explain discrepancy between studies. In particular, various choice of ROIs among studies (i.e. selection of structure from only contiguous voxels with the highest values to the entire structure) hampers comparisons between studies. For instance, the difference of FA in the genu of the CC between 0.44 in [55] and 0.80 in [54], may be largely due to the ROI used for measurement. Therefore it is important to ensure the DT- and KT-derived indices are reliable across raters within-group, as ROIbased measurement often required rater decisions which may have impacted ROI placement between observers. Our results show that ICC values are near 1 for various ROIs over all metrics, indicating high reliability of ROIbased measurement performed in the brain. It should be also noted that regional variability of DTI values between publications may relate to age differences within-group aside from selection of ROIs, acquisition parameters and SNR. Considerable inter-subject variability of DTI parameters has been shown in previous studies that mostly reported the age dependence of DTI metrics [8789]. Our subjects were young adults (mean age $24 \pm 2$ ) and this may contribute on variability of diffusion metrics as compared to those in the literature.

In the spinal cord, there are numerous reports in the literature available for comparison with our results. In order to ensure the similarity of the acquisition sequences and parameters, here we focus on comparing our DT-derived values with those by Qian et al. [64] (a study that our raw data were originally obtained) and Samson et al. [27] (a multi-centre study with the compatible acquisition protocols as ours). Our FA values with Rician denoising are found to be significantly lower than those previous reported by Qian et al. $(0.81-0.84 ; p<0.05)$ while our MD, $\mathrm{AD}$ and $\mathrm{RD}$ values with Rician denoising are not significantly different from those from the same study [64]. This suggests that Rician NLM filtering might reduce overestimation of FA in the spinal cord, which is common observed in DTI of the brain $[68,85,90]$, by decreasing estimation 
error. Our column specific measurements of MD are at the lower end of the previously reported range of 0.93$1.29 \mathrm{~mm}^{2} / \mathrm{ms}$ by Samson et al. [27] while FA measurements are higher than the range measured by the same study (0.59-0.63). Our RD values are lower than the range measured by Samson et al. $\left(0.68-0.84 \mathrm{~mm}^{2} / \mathrm{ms}\right)$ [27] while $A D$ values are at the higher end of the previously reported range of $1.43-2.22 \mathrm{~mm}^{2} / \mathrm{ms}$ by the same study. Overall, DTI metrics are likely to be within similar ranges, however difference among protocols and the associated SNR in each study must be taken into account when comparing between those DTI metrics.

\section{Consideration for clinical applications}

It is worth noting that the DKI data acquisition took around 20 min per person, because we obtained whole brain DKI data with 2 averages in order to evaluate quality of different processing methods through a whole brain with reasonable SNR. Our results showed significant decrease of $\chi^{2}$ values, indicating quality of DKI-derived maps improved through a whole brain within reasonable scan time when CLLS-R estimation was performed. Thus, by reducing the number of slices carefully selected for areas of interest, neurology- or neuroscience-related application studies should be feasible, which would last a clinically acceptable time frame (less than $10 \mathrm{~min}$ ). Additionally, Rician NLM denoising in combination with CLLS can be readily implemented as it is based on a LSE algorithm available through existing commercial programs. Therefore, practical use of the combined Rician denoising method is widely expected in characterizing microarchitectural integrity of normal and pathological states.

\section{Conclusions}

We have demonstrated that a Rician denoising filter incorporated with CLLS (CLLS-R) significantly increases SNR while reducing estimation errors. Our results suggest that the combined postprocessing method may provide the capability to more reliably quantify tissue properties both in the brain and the spinal cord with adequate SNR levels while it is easy to implement. Future studies are warranted towards investigating clinical and neuroscientific applications using this method.

\section{Supplementary Information}

The online version contains supplementary material available at https://doi. org/10.1186/s12880-021-00549-9.

Additional file 1. Supporting Information Table S1 showing comparison for DT- and KT-derived metrics between CLLS and CLLS-R analyses.

\section{Abbreviations}

dMRI: Diffusion magnetic resonance imaging; DTI: Diffusion tensor imaging; WM: White matter; DKI: Diffusion kurtosis imaging; GM: Gray matter; FA: Fractional anisotropy; MD: Mean diffusivity; RD: Radial diffusivity; AD: Axial diffusivity; MK: Mean kurtosis; RK: Radial kurtosis; AK: Axial kurtosis; SNR: Signalto-noise ratio; NLM: Nonlocal means; CLLS: Constrained linear least squares; CLLS-R: Constrained linear least squares with Rician denoising; LS: Leastsquares; EPI: Echo-planar-imaging; AIR: Automated image registration; ROIs: Regions of interest; PUT: Putamen; GP: Globus pallidus; CC: Corpus callosum; IC: Internal capsule; EC: External capsule; Cg: Cingulum; $X^{2}$ : Chi-square; CSF: Cerebrospinal fluid; ICC: Intraclass correlation coefficient.

\section{Acknowledgements}

The authors would like to thank Dr. Marco Battiston for constructive comments.

\section{Authors' contributions}

Study concepts and design: ZZ and MK; literature research and data collection: ZZ, DV, WQ and MK; statistical analysis: ZZ and DV; manuscript preparation: ZZ and DV; manuscript editing:WQ and MK. All authors have read and approved the manuscript.

\section{Funding}

This work was supported by a grant from the University of Hong Kong funding program for basic research. Funding was utilized for data acquisition.

\section{Availability of data and materials}

All data are fully available upon reasonable request. The corresponding author should be contacted if someone wants to request the data.

\section{Ethics approval and consent to participate}

This study was approved by the University of Hong Kong Institutional Review Board (IRB) and all subjects provided the signed consent.

\section{Consent for publication}

Not applicable.

\section{Competing interests}

The authors declare no competing interests.

\section{Author details}

${ }^{1}$ Department of Diagnostic Radiology, The University of Hong Kong, Hong Kong SAR, China. ${ }^{2}$ Department of Neuroinflammation, Faculty of Brain Sciences, UCL Queen Square Institute of Neurology, London, UK. ${ }^{3}$ Present Address: Philips Healthcare, Shanghai, China. ${ }^{4}$ Present Address: Laboratory of Clinical Investigation, National Institute on Aging, NIH, Baltimore, USA.

Received: 14 May 2020 Accepted: 18 January 2021

Published online: 30 January 2021

\section{References}

1. Basser PJ, Mattiello J, LeBihan D. MR diffusion tensor spectroscopy and imaging. Biophys J. 1994;66(1):259-67.

2. Pierpaoli C, Jezzard P, Basser PJ, Barnett A, Di Chiro G. Diffusion tensor MR imaging of the human brain. Radiology. 1996;201(3):637-48.

3. Le Bihan D, Mangin JF, Poupon C, Clark CA, Pappata S, Molko N, Chabriat H. Diffusion tensor imaging: concepts and applications. J Magn Reson Imaging. 2001;13(4):534-46.

4. Poupon C, Mangin J, Clark CA, Frouin V, Regis J, Le Bihan D, Bloch I. Towards inference of human brain connectivity from MR diffusion tensor data. Med Image Anal. 2001;5(1):1-15.

5. Mori S, Zhang J. Principles of diffusion tensor imaging and its applications to basic neuroscience research. Neuron. 2006;51(5):527-39.

6. Mori S, Barker PB. Diffusion magnetic resonance imaging: its principle and applications. Anat Rec. 1999;257(3):102-9.

7. Tae WS, Ham BJ, Pyun SB, Kang SH, Kim BJ. Current clinical applications of diffusion-tensor imaging in neurological disorders. J Clin Neurol. 2018;14(2):129-40. 
8. Moura LM, Luccas R, de Paiva JPQ, Amaro E Jr, Leemans A, Leite CDC, Otaduy MCG, Conforto AB. Diffusion tensor imaging biomarkers to predict motor outcomes in stroke: a narrative review. Front Neurol. 2019;10:445.

9. Alexander AL, Lee JE, Lazar M, Field AS. Diffusion tensor imaging of the brain. Neurotherapeutics. 2007;4(3):316-29.

10. Basser PJ, Pierpaoli C. Microstructural and physiological features of tissues elucidated by quantitative-diffusion-tensor MRI. J Magn Reson B. 1996;111(3):209-19.

11. Pierpaoli C, Basser PJ. Toward a quantitative assessment of diffusion anisotropy. Magn Reson Med. 1996;36(6):893-906.

12. Jensen JH, Helpern JA, Ramani A, Lu H, Kaczynski K. Diffusional kurtosis imaging: the quantification of non-gaussian water diffusion by means of magnetic resonance imaging. Magn Reson Med. 2005;53(6):1432-40.

13. Lu H, Jensen JH, Ramani A, Helpern JA. Three-dimensional characterization of non-gaussian water diffusion in humans using diffusion kurtosis imaging. NMR Biomed. 2006;19(2):236-47.

14. Hui ES, Cheung MM, Qi L, Wu EX. Towards better MR characterization of neural tissues using directional diffusion kurtosis analysis. Neuroimage. 2008:42(1):122-34.

15. Jensen JH, Falangola MF, Hu C, Tabesh A, Rapalino O, Lo C, Helpern JA. Preliminary observations of increased diffusional kurtosis in human brain following recent cerebral infarction. NMR Biomed. 2010;24:452.

16. Helpern JA, Adisetiyo V, Falangola MF, Hu C, Di Martino A, Williams K, Castellanos FX, Jensen JH. Preliminary evidence of altered gray and white matter microstructural development in the frontal lobe of adolescents with attention-deficit hyperactivity disorder: a diffusional kurtosis imaging study. J Magn Reson Imaging. 2011;33(1):17-23.

17. Falangola MFJJ, Babb JS, Hu C, Castellanos FX, Di Martino A, Ferris SH, Helpern JA. Age-related non-Gaussian diffusion patterns in the prefrontal brain. J Magn Reson Imaging. 2008;28(6):1345-50.

18. Cheung MM, Hui ES, Chan KC, Helpern JA, Qi L, Wu EX. Does diffusion kurtosis imaging lead to better neural tissue characterization? A rodent brain maturation study. Neuroimage. 2009;45(2):386-92.

19. Van Cauter S, Veraart J, Sijbers J, Peeters RR, Himmelreich U, De Keyzer F, Van Gool SW, Van Calenbergh F, De Vleeschouwer S, Van Hecke W, Sunaert S. Gliomas: diffusion kurtosis MR imaging in grading. Radiology. 2012;263(2):492-501.

20. Manjon JV, Carbonell-Caballero J, Lull JJ, Garcia-Marti G, Marti-Bonmati L, Robles M. MRI denoising using non-local means. Med Image Anal. 2008;12(4):514-23.

21. Aja-Fernandez S, Niethammer M, Kubicki M, Shenton ME, Westin CF. Restoration of DWI data using a Rician LMMSE estimator. IEEE Trans Med Imaging. 2008;27(10):1389-403.

22. Skare S, Li T, Nordell B, Ingvar M. Noise considerations in the determination of diffusion tensor anisotropy. Magn Reson Imaging. 2000;18(6):659-69.

23. Gudbjartsson $\mathrm{H}$, Patz S. The Rician distribution of noisy MRI data. Magn Reson Med. 1995;34(6):910-4.

24. Rossi C, Boss A, Steidle G, Martirosian P, Klose U, Capuani S, Maraviglia B, Claussen CD, Schick F. Water diffusion anisotropy in white and gray matter of the human spinal cord. J Magn Reson Imaging. 2008;27(3):476-82.

25. Zhang BT, Li M, Yu LL, Dai YM, Yu SN, Jiang JL. Diffusion tensor imaging of spinal microstructure in healthy adults: improved resolution with the readout segmentation of long variable echo-trains. Neural Regen Res. 2017;12(12):2067-70.

26. Ries M, Jones RA, Dousset $\mathrm{V}$, Moonen CT. Diffusion tensor MRI of the spinal cord. Magn Reson Med. 2000;44(6):884-92.

27. Samson RS, Levy S, Schneider T, Smith AK, Smith SA, Cohen-Adad J, Gandini Wheeler-Kingshott CA. ZOOM or Non-ZOOM? Assessing spinal cord diffusion tensor imaging protocols for multi-centre studies. PLOS ONE. 2016;11(5):e0155557.

28. Vedantam A, Jirjis MB, Schmit BD, Wang MC, Ulmer JL, Kurpad SN. Characterization and limitations of diffusion tensor imaging metrics in the cervical spinal cord in neurologically intact subjects. J Magn Reson Imaging. 2013;38(4):861-7.

29. Hendrix P, Griessenauer CJ, Cohen-Adad J, Rajasekaran S, Cauley KA, Shoja MM, Pezeshk P, Tubbs RS. Spinal diffusion tensor imaging: a comprehensive review with emphasis on spinal cord anatomy and clinical applications. Clin Anat. 2015;28(1):88-95.
30. Falangola MF, Jensen JH, Babb JS, Hu C, Castellanos FX, Di Martino A, Ferris SH, Helpern JA. Age-related non-Gaussian diffusion patterns in the prefrontal brain. J Magn Reson Imaging. 2008;28(6):1345-50.

31. Brion V, Poupon C, Riff O, Aja-Fernandez S, Tristan-Vega A, Mangin JF, Le Bihan D, Poupon F. Parallel MRI noise correction: an extension of the LMMSE to non central chi distributions. Med Image Comput Comput Assist Interv. 2011;14(Pt 2):226-33.

32. Parker GJ, Schnabel JA, Symms MR, Werring DJ, Barker GJ. Nonlinear smoothing for reduction of systematic and random errors in diffusion tensor imaging. J Magn Reson Imaging. 2000;11 (6):702-10.

33. Tabelow K, Polzehl J, Spokoiny V, Voss HU. Diffusion tensor imaging: structural adaptive smoothing. Neuroimage. 2008;39(4):1763-73.

34. Ding Z, Gore JC, Anderson AW. Reduction of noise in diffusion tensor images using anisotropic smoothing. Magn Reson Med. 2005;53(2):485-90.

35. Veraart J, Novikov DS, Christiaens D, Ades-Aron B, Sijbers J, Fieremans E. Denoising of diffusion MRI using random matrix theory. Neuroimage. 2016;142:394-406.

36. Becker SM, Tabelow K, Voss HU, Anwander A, Heidemann RM, Polzehl J. Position-orientation adaptive smoothing of diffusion weighted magnetic resonance data (POAS). Med Image Anal. 2012;16(6):1142-55.

37. Wiest-Daessle N, Prima S, Coupe P, Morrissey SP, Barillot C. Non-local means variants for denoising of diffusion-weighted and diffusion tensor MRI. Med Image Comput Comput Assist Interv. 2007;10(Pt 2):344-51.

38. Manjon JV, Coupe P, Marti-Bonmati L, Collins DL, Robles M. Adaptive nonlocal means denoising of MR images with spatially varying noise levels. J Magn Reson Imaging. 2010;31(1):192-203.

39. Coupe P, Yger P, Prima S, Hellier P, Kervrann C, Barillot C. An optimized blockwise nonlocal means denoising filter for 3-D magnetic resonance images. IEEE Trans Med Imaging. 2008;27(4):425-41.

40. Wiest-Daessle N, Prima S, Coupe P, Morrissey SP, Barillot C. Rician noise removal by non-Local Means filtering for low signal-to-noise ratio MRI: applications to DT-MRI. Medical image computing and computerassisted intervention : MICCAI International Conference on Medical Image Computing and Computer-Assisted Intervention 2008; 1 (Pt 2):171-179.

41. Coupe PYP, Prima S, Hellier P, Kervrann C, Barillot C. An optimized blockwise nonlocal means denoising filter for 3-D magnetic resonance images. IEEE Trans Med Imaging. 2008;27(4):425-41.

42. Wu X, Liu S, Wu M, Sun H, Zhou J, Gong Q, Ding Z. Nonlocal denoising using anisotropic structure tensor for 3D MRI. Med Phys. 2013;40(10):101904

43. Manjon JV, Coupe P, Concha L, Buades A, Collins DL, Robles M. Diffusion weighted image denoising using overcomplete local PCA. PLoS ONE. 2013;8(9):e73021.

44. Wiest-Daesslé NPS, Coupé P, Morrissey SP, Barillot C. Rician noise removal by non-Local Means filtering for low signal-to-noise ratio MRI: applications to DT-MRI. Med Image Comput Comput Assist Interv. 2008;11(pt 2):171-9.

45. Zhou MX, Yan X, Xie HB, Zheng H, Xu D, Yang G. Evaluation of non-local means based denoising filters for diffusion kurtosis imaging using a new phantom. PLoS ONE. 2015;10(2):e0116986.

46. Veraart J, Van Hecke W, Sijbers J. Constrained maximum likelihood estimation of the diffusion kurtosis tensor using a Rician noise model. Magn Reson Med. 2011;66(3):678-86.

47. Andersson JL. Maximum a posteriori estimation of diffusion tensor parameters using a Rician noise model: why, how and but. Neuroimage. 2008:42(4):1340-56.

48. Basu S, Fletcher T, Whitaker R. Rician noise removal in diffusion tensor MRI. Med Image Comput Comput Assist Interv. 2006;9(Pt 1):117-25.

49. Descoteaux M, Wiest-Daessle N, Prima S, Barillot C, Deriche R. Impact of Rician adapted Non-Local Means filtering on HARDI. Medical image computing and computer-assisted intervention : MICCAI International Conference on Medical Image Computing and Computer-Assisted Intervention 2008;11(Pt 2):122-130

50. Reischauer C, Gutzeit A. Image denoising substantially improves accuracy and precision of intravoxel incoherent motion parameter estimates. PLOS ONE. 2017;12(4):e0175106.

51. Gonzalez JE, Thompson PM, Zhao A, Tu Z. Modeling diffusion-weighted $\mathrm{MRI}$ as a spatially variant gaussian mixture: application to image denoising. Med Phys. 2011;38(7):4350-64. 
52. Tabesh A, Jensen JH, Ardekani BA, Helpern JA. Estimation of tensors and tensor-derived measures in diffusional kurtosis imaging. Magn Reson Med. 2011;65(3):823-36.

53. Su B, Liu Q, Chen J, Wu X. Non-local mean denoising in diffusion tensor space. Exp Ther Med. 2014;8(2):447-53.

54. Latt J, Nilsson M, Wirestam R, Stahlberg F, Karlsson N, Johansson M, Sundgren $P$, van Westen D. Regional values of diffusion kurtosis estimates in the healthy brain. J Magn Reson Imaging. 2013;37:610-8.

55. Das SK, Wang JL, Bing L, Bhetuwal A, Yang HF. Regional Values of Diffusional Kurtosis Estimates in the Healthy Brain during Normal Aging. Clin Neuroradiol. 2017;27(3):283-98.

56. Andre ED, Grinberg F, Farrher E, Maximov II, Shah NJ, Meyer C, Jaspar M, Muto V, Phillips C, Balteau E. Influence of noise correction on intra- and inter-subject variability of quantitative metrics in diffusion kurtosis imaging. PLoS ONE. 2014;9(4):e94531.

57. Tu TW, Budde MD, Xie M, Chen YJ, Wang Q, Quirk JD, Song SK. Phasealigned multiple spin-echo averaging: a simple way to improve signalto-noise ratio of in vivo mouse spinal cord diffusion tensor image. Magn Reson Imaging. 2014;32(10):1335-43.

58. Wheeler-Kingshott CA, Hickman SJ, Parker GJ, Ciccarelli O, Symms MR, Miller DH, Barker GJ. Investigating cervical spinal cord structure using axial diffusion tensor imaging. Neuroimage. 2002;16(1):93-102.

59. Saritas EU, Cunningham CH, Lee JH, Han ET, Nishimura DG. DWI of the spinal cord with reduced FOV single-shot EPI. Magn Reson Med. 2008;60(2):468-73.

60. Cohen-Adad J, El Mendili MM, Lehericy S, Pradat PF, Blancho S, Rossignol $\mathrm{S}, \mathrm{Benali} \mathrm{H}$. Demyelination and degeneration in the injured human spinal cord detected with diffusion and magnetization transfer MRI. Neuroimage. 2011;55(3):1024-33.

61. Xu J, Shimony JS, Klawiter EC, Snyder AZ, Trinkaus K, Naismith RT, Benzinger TL, Cross AH, Song SK. Improved in vivo diffusion tensor imaging of human cervical spinal cord. Neuroimage. 2013;67:64-76.

62. Liu C, Bammer R, Acar B, Moseley ME. Characterizing non-Gaussian diffusion by using generalized diffusion tensors. Magn Reson Med. 2004;51(5):924-37.

63. Veraart J, Sijbers J, Sunaert S, Leemans A, Jeurissen B. Weighted linear least squares estimation of diffusion MRI parameters: strengths, limitations, and pitfalls. Neuroimage. 2013;81:335-46.

64. Qian W, Chan Q, Mak H, Zhang Z, Anthony MP, Yau KK, Khong PL, Chan $\mathrm{KH}$, Kim M. Quantitative assessment of the cervical spinal cord damage in neuromyelitis optica using diffusion tensor imaging at 3 Tesla. J Magn Reson Imaging. 2011:33(6):1312-20.

65. Glenn GR, Tabesh A, Jensen JH. A simple noise correction scheme for diffusional kurtosis imaging. Magn Reson Imaging. 2015;33(1):124-33.

66. Jensen JH, Falangola MF, Hu C, Tabesh A, Rapalino O, Lo C, Helpern JA. Preliminary observations of increased diffusional kurtosis in human brain following recent cerebral infarction. NMR Biomed. 2011;24(5):452-7.

67. Woods RP, Grafton ST, Holmes CJ, Cherry SR, Mazziotta JC. Automated image registration: I. General methods and intrasubject, intramodality validation. J Comput Assist Tomogr. 1998;22(1):139-52.

68. Farrell JA, Landman BA, Jones CK, Smith SA, Prince JL, van Zijl PC, Mori S. Effects of signal-to-noise ratio on the accuracy and reproducibility of diffusion tensor imaging-derived fractional anisotropy, mean diffusivity, and principal eigenvector measurements at 1.5 T. J Magn Reson Imaging. 2007;26(3):756-67.

69. Tamraz JC, Comair Y. Atlas of Regional Anatomy of the Brain Using MRI: With Functional Correlations. Berlin: Springer; 2000.

70. Shrout PE, Fleiss JL. Intraclass correlations: uses in assessing rater reliability. Psychol Bull. 1979;86(2):420-8.

71. Wang ZJ, Chia JM, Ahmed S, Rollins NK. Signal-to-noise assessment for diffusion tensor imaging with single data set and validation using a difference image method with data from a multicenter study. Med Phys. 2014;41(9):092302.

72. Anderson AW. Theoretical analysis of the effects of noise on diffusion tensor imaging. Magn Reson Med. 2001;46(6):1174-88.
73. Koay CG, Carew JD, Alexander AL, Basser PJ, Meyerand ME. Investigation of anomalous estimates of tensor-derived quantities in diffusion tensor imaging. Magn Reson Med. 2006:55(4):930-6.

74. Seo Y, Wang ZJ, Morriss MC, Rollins NK. Minimum SNR and acquisition for bias-free estimation of fractional anisotropy in diffusion tensor imaging: a comparison of two analytical techniques and field strengths. Magn Reson Imaging. 2012;30(8):1123-33.

75. Dietrich O, Raya JG, Reeder SB, Reiser MF, Schoenberg SO. Measurement of signal-to-noise ratios in MR images: influence of multichannel coils, parallel imaging, and reconstruction filters. J Magn Reson Imaging. 2007;26(2):375-85

76. Goerner FL, Clarke GD. Measuring signal-to-noise ratio in partially parallel imaging MRI. Med Phys. 2011;38(9):5049-57.

77. Lin FH, Chen YJ, Belliveau JW, Wald LL. A wavelet-based approximation of surface coil sensitivity profiles for correction of image intensity inhomogeneity and parallel imaging reconstruction. Hum Brain Mapp. 2003;19(2):96-111.

78. Hiratsuka Y, Miki H, Kikuchi K, Kiriyama I, Mochizuki T, Takahashi S, Sadamoto K. Sensitivity of an eight-element phased array coil in 3 Tesla MR imaging: a basic analysis. Magn Reson Med Sci. 2007:6(3):177-81.

79. Roemer PB, Edelstein WA, Hayes CE, Souza SP, Mueller OM. The NMR phased array. Magn Reson Med. 1990;16(2):192-225.

80. Fillard P, Pennec X, Arsigny V, Ayache N. Clinical DT-MRI estimation, smoothing, and fiber tracking with log-Euclidean metrics. IEEE Trans Med Imaging. 2007;26(11):1472-82.

81. Martin-Fernandez M, Munoz-Moreno E, Cammoun L, Thiran JP, Westin CF, Alberola-Lopez C. Sequential anisotropic multichannel Wiener filtering with Rician bias correction applied to 3D regularization of DWI data. Med Image Anal. 2009;13(1):19-35.

82. Jeong EK, Kim SE, Guo J, Kholmovski EG, Parker DL. High-resolution DTI with 2D interleaved multislice reduced FOV single-shot diffusionweighted EPI (2D ss-rFOV-DWEPI). Magn Reson Med. 2005;54(6):1575-9.

83. Benitez A, Jensen JH, Falangola MF, Nietert PJ, Helpern JA. Modeling white matter tract integrity in aging with diffusional kurtosis imaging. Neurobiol Aging. 2018;70:265-75.

84. Jensen JH, Helpern JA. MRI quantification of non-Gaussian water diffusion by kurtosis analysis. NMR Biomed. 2010;23(7):698-710.

85. Seo Y, Rollins NK, Wang ZJ. Reduction of bias in the evaluation of fractional anisotropy and mean diffusivity in magnetic resonance diffusion tensor imaging using region-of-interest methodology. Sci Rep. 2019;9(1):13095.

86. Szczepankiewicz F, Latt J, Wirestam R, Leemans A, Sundgren P, van Westen D, Stahlberg F, Nilsson M. Variability in diffusion kurtosis imaging: impact on study design, statistical power and interpretation. Neuroimage. 2013;76:145-54.

87. Lebel C, Walker L, Leemans A, Phillips L, Beaulieu C. Microstructural maturation of the human brain from childhood to adulthood. Neuroimage. 2008;40(3):1044-55.

88. Schneiderman JS, Buchsbaum MS, Haznedar MM, Hazlett EA, Brickman AM, Shihabuddin L, Brand JG, Torosjan Y, Newmark RE, Tang C, Aronowitz J. Paul-Odouard R, Byne W, Hof PR. Diffusion tensor anisotropy in adolescents and adults. Neuropsychobiology. 2007;55(2):96-111.

89. Lebel C, Gee M, Camicioli R, Wieler M, Martin W, Beaulieu C. Diffusion tensor imaging of white matter tract evolution over the lifespan. Neuroimage. 2012;60(1):340-52.

90. Polders DL, Leemans A, Hendrikse J, Donahue MJ, Luijten PR, Hoogduin $J M$. Signal to noise ratio and uncertainty in diffusion tensor imaging at 1.5, 3.0, and 7.0 Tesla. J Magn Reson Imaging. 2011;33(6):1456-63.

\section{Publisher's Note}

Springer Nature remains neutral with regard to jurisdictional claims in published maps and institutional affiliations. 